10 Minnaert, M., Nature, 165, 663-664 (1950).

11 See for example Burke, J. J., J. opt. Soc. Am., 60, 1262-1264 (1970).

12 Prokhorov, A. M., Bunkin, F. V., Gochelashvily, K. S., and Shishov, V. I. Proc. IEEE, 63, 790-811 (1975)

13 Bramley, E. N., and Young, M., Proc. IEE, Lond, 114, 553-556 (1967).

14 Taylor, L. S., and Infosino, C. J., J. opt. Soc. Am., 65, 78-84 (1975)

15 Jakeman, E. and McWhirter, J. G. J Phys. A., 9, 785-797 (1976)

16 Mikesell, A. H., Hoag, A. A., and Hall, J. S., J. opt. Soc. Am., 41, 689-695 (1951).

\section{$Q$ in cosmology}

THE following example in classical mechanics yields the key equations of relativistic cosmology, including the new equations by Harrison ${ }^{1}$. The argument given here develops that of an earlier note ${ }^{2}$.

A set of $n$ gravitating particles of masses $m_{i}$ are at positions $\mathbf{r}_{t}(0)=\mathbf{a}_{i}$ relative to a fixed origin at the time $t=0$, when an explosion gives a radial motion $\mathbf{r}_{i}(t)=R(t) \mathbf{a}_{i}$ to all the particles. The scale factor $R$ is the same for all the particles. The kinetic energy of the system at time $t$ is a sum of terms of the type $\frac{1}{2} m_{t} v_{l}^{2}$, and the gravitational potential energy is a sum of terms of the form $\left(\left|\mathbf{a}_{i}-\mathbf{a}_{j}\right|=\mathbf{a}_{i j}\right)$

$$
-G \frac{m_{i} m_{f}}{r_{i j}(t)}=-G \frac{m_{i} m_{j}}{a_{i j}} \frac{1}{R(t)}
$$

Accordingly, the energy equation of the system is

$$
E=A \dot{R}^{2}-B / R, A \equiv \frac{1}{2} \sum_{i} m_{i} a_{i}{ }^{2}, B \equiv \frac{G}{2} \sum_{i \neq j} \frac{m_{i} m_{j}}{a_{i j}}
$$

To attain a formal analogy with relativistic cosmology, write

$$
E / A \equiv-k c^{2}, B / A \equiv C,
$$

and add a cosmological energy term $A \lambda R^{2} / 3$. This gives the following energy equation and its first and second time derivatives

$$
\begin{array}{ll}
\frac{\lambda}{3}=H^{2}+\frac{k c^{2}}{R^{2}}-\frac{C}{R^{3}} & (H \equiv \dot{R} / R) \\
\frac{\lambda}{3}=\frac{C}{2 R^{3}}-q H^{2} & \left(q \equiv-\ddot{R} R / \dot{R}^{2}\right) \\
\frac{\lambda}{3}=Q H^{2}-\frac{C}{R^{3}} & \left(Q \equiv R^{2} \dddot{R} / \dot{R}^{3}\right)
\end{array}
$$

The importance of the $Q$ parameter has already been noted ${ }^{\mathbf{1}}$. One obtains the basic cosmological differential equation in two forms which are independent of the experimentally little known parameter $\lambda$, by equating equations (2) and (3) or by equating (2) and (4)

$$
\begin{gathered}
\frac{k c^{2}}{R^{2}}=\frac{3 C}{2 R^{3}}-(q+1) H^{2} \\
\frac{k c^{2}}{R^{2}}=(Q-1) H^{2}
\end{gathered}
$$

Adding twice (3) to (4) and taking (3) from (4):

$$
\lambda=(Q-2 q) H^{2}, \frac{3 C}{2 R}=(Q+q) H^{2}
$$

In a Newtonian context, the system is ever expanding if $E$ is positive; that is, if $k$ is negative. By equation (6), the appropriate condition is $Q<1$, and for $\lambda=0$ models, it is equivalent to $q<\frac{1}{2}$ (equation (7)). The inequalities are reversed if the system is to collapse again. Note that only if one puts ( $\rho$ is the mass density)

$$
C=\frac{8 \pi}{3} G \rho R^{3}
$$

do these become the equations given by Harrison ${ }^{1}$. The simplicity of the example given here is illuminating: the role of $q$, which determines whether a $(\lambda \rightleftharpoons 0)$-model is closed or open, can clearly be taken over by $Q$ if $\lambda \neq 0$. A continuous distribution of gravitating matter, which is needed to obtain equation (9) in general relativity, and which was used in ref. 1, is clearly not needed here. A discrete distribution of galaxies with sufficient symmetry to ensure the continued validity of Hubble's Law is all that is required. It is to be emphasised that in Newtonian cosmology it is possible to give discussions of interacting particles and discrete matter distributions which give Hubble's Law, though the corresponding general relativistic problems remain unsolved.

This possibility has been noted already ${ }^{2,3}$, but no example has been given before. The simplest distribution to verify this possibility is a set of eight galaxies, each of mass $m$, on the vertices of a cube of side $2 a_{0}$, which are given velocities, $v$, radially away from the centre of the cube. Because of the seven other galaxies each galaxy is then subject to a deceleration in the direction of the centre. Its magnitude is

$$
\frac{D}{2 a(t)^{2}}, D \equiv\left[1+\frac{\sqrt{ } 2}{2}+\frac{\sqrt{ } 3}{9}\right] \frac{G m}{2}
$$

where $2 a(t)$ is the side of the cube at time $t$. If the escape velocity $v_{\mathrm{esc}}=\sqrt{ }\left(D / a_{0}\right)$ has been exceeded, the Hubble parameter is

$$
\begin{aligned}
\frac{\theta^{3}\left(1+w^{2}\right)^{\frac{1}{2}}}{D w^{3}}, & \theta \equiv\left(v^{2}-v_{\mathrm{esc}}^{2}\right)^{\frac{1}{2}} \\
& w \equiv \theta \sqrt{ }[a(t) / D] .
\end{aligned}
$$

Essentially, a topic for so far undiscovered theorems in Newtonian physics is raised here: the classification of (usually unstable) particle configurations which, for a given interaction, are consistent with a Hubble type expansion; and expressions for the Hubble parameter should also be found in each case.

Department of Mathematics,

P. T. LANDSBERG

The University,

Southampton SO9 $5 \mathrm{NH}, U K$

Received April 28; accepted August 4, 1976.

1 Harrison, E. R., Nature, 260, 591 (1976).

2 Landsberg, P. T., Nature, 242, 104 (1973).

3 Landsberg, P. T., Nature, 244, 66 (1973).

\section{Anomalous $M_{1}$ tide at Lagos}

I HAVE recently described ${ }^{1}$ the phenomenal appearance in the seas off western Europe of a tidal component with a period of exactly one mean lunar day, namely the " $M_{1}$ tide". Although only a few $\mathrm{mm}$ in amplitude, it is about ten times larger than one would expect from comparison with ordinary diurnal tides, and suggests a resonant response of 